\title{
Toward the development of a fully elastic mitral ring: Preliminary, acute, in vivo evaluation of physiomechanical behavior
}

\author{
Paolo Ferrazzi, MD, FETCS, ${ }^{\mathrm{a}}$ Attilio Iacovoni, MD, ${ }^{\mathrm{a}}$ Samuele Pentiricci, MD, ${ }^{\mathrm{a}}$ Michele Senni, MD, FESC, ${ }^{\mathrm{a}}$ Maria Iascone, BSc, \\ $\mathrm{PhD},{ }^{\mathrm{b}}$ Nicolas Borenstein, DVM, PhD, ${ }^{\mathrm{c}}$ Luc Behr, DVM, PhD,${ }^{\mathrm{c}}$ Alessandro Borghi, MEng, PhD, ${ }^{\mathrm{d}}$ Rossella Balossino, MEng, \\ $\mathrm{PhD}^{\mathrm{e}}$, and Eugenio Quaini, $\mathrm{MD}^{\mathrm{a}}$
}

\begin{abstract}
Objectives: The optimal repair of functional mitral regurgitation is still debated. No device is able to simultaneously abolish mitral regurgitation and replicate natural mitral annular dynamics. We have tested a fully elastic mitral ring in an acute animal study with the purpose of evaluating (1) ring design and implantation technique, (2) elastic performance, and (3) acute effects on the native mitral annulus.
\end{abstract}

\begin{abstract}
Methods: Ten healthy sheep underwent surgical implantation of mitral devices, the elastic component of which is represented by a helicoid metallic spring. Preimplantation and postimplantation echocardiographic parameter measurements to evaluate annular dynamics and ventricular function comprise mitral annular motion, systolic tissue Doppler imaging peak wave, transmitral pressure gradient, peak transmitral flow velocity, and ejection fraction. Postimplantation angiographic analysis allowed measurement of the mitral annular area and perimeter variations by means of segmentation of the radiopaque mitral device contour.
\end{abstract}

Results: No significant difference in terms of ejection fraction $(P=.13)$ and systolic tissue Doppler imaging peak wave $(P=.87)$ was found before and after implantation. Mitral annular motion $(1.16 \mathrm{~cm})$ was preserved. The percentage of systolic annular reduction derived from angiographic analysis was $14.1 \%$ (range, $7.7 \%-$ $19.7 \%$ ) in terms of area and $7.2 \%$ (range, $4.9 \%-10.0 \%$ ) in terms of perimeter.

Conclusions: A mitral elastic ring, implantable by using a standard technique, acutely preserves mitral annular dynamics, allowing area and perimeter changes. Further chronic study is needed to verify the biocompatibility and durability of the device.

The mitral annulus (MA) is a discontinuous fibromuscular ring with a rough anatomic structure and complex physiology. It is a dynamic structure that undergoes changes in size and shape throughout the cardiac cycle. ${ }^{1}$

The change in the size of the annular area has been demonstrated in animal and human models, and a cyclic reduction of annular area has been revealed, with minimal area in systole. ${ }^{2-5}$ It has also been pointed out that the shape of the MA is a hyperbolic paraboloid and is therefore reminiscent of a saddle, with a higher curvature in systole. ${ }^{6}$ Furthermore, the annulus is seen to move during the cardiac cycle; it undergoes anterior displacement toward the apex during ventricular systole and posterior displacement toward the base during ventricular diastole, as well as during atrial contraction. $^{7,8}$

From Dipartimento Cardiovascolare Clinico e di Ricerca ${ }^{\mathrm{a}}$ and Dipartimento di Medicina di Laboratorio, ${ }^{\mathrm{b}}$ Ospedali Riuniti, Bergamo, Italy; IMM Recherche, ${ }^{\mathrm{c}}$ Paris, France; Department of Chemical Engineering, ${ }^{\mathrm{d}}$ Imperial College London, London, United Kingdom; and Laboratorio di Meccanica delle Strutture Biologiche, ${ }^{\mathrm{e}}$ Politecnico di Milano, Milano, Italy.

Supported by MSA Mollificio S.Ambrogio s.p.a. and Ab Medica s.p.a.

Received for publication May 15, 2008; revisions received Aug 18, 2008; accepted for publication Aug 27, 2008.

Address for reprints: Paolo Ferrazzi, MD, FETCS, Cardiac Surgery Unit, Dipartimento Cardiovascolare Clinico e di Ricerca, Ospedali Riuniti, Largo Barozzi, 1, 24128

Bergamo, Italy (E-mail: pferrazzi@ospedaliriuniti.bergamo.it).

J Thorac Cardiovasc Surg 2009;137:174-9

$0022-5223 / \$ 36.00$

Copyright (c) 2009 by The American Association for Thoracic Surgery

doi:10.1016/j.jtcvs.2008.08.041
The MA dynamics are regulated by the elastic properties of the myocardial tissue. ${ }^{7}$ Theoretically, the ideal annuloplasty ring design for functional mitral regurgitation (FMR) should, in addition to permanently abolishing mitral insufficiency, promote an MA dynamic motion, facilitate its sphincteric contraction, and provide an active force to help in the orifice's systolic mitral area reduction. A fully elastic mitral ring prototype was developed and implanted on the MAs of 10 healthy sheep to test this hypothesis. The purpose of this study was to acutely assess the maintenance of the physiologic annular motion behavior.

\section{MATERIALS AND METHODS}

The animal study was designed to evaluate immediate (day 0 ) and longterm (3-6 months) results after implantation of the elastic device in healthy sheep. Long-term evaluation will mostly aim at the device's physical performance and biocompatibility assessment. The present study reports the early results, with the aim of demonstrating the feasibility of the procedure and the device's mechanical performance in its interaction with the native MA.

\section{Mitral Device}

The device used for this study is a fully elastic and flexible ring that is able to expand and bend according to MA dynamics. By definition, this device follows the law of elasticity, which states that an elastic body deforms under external forces but then returns to its original shape and dimensions when the external forces are removed.

The device is made up of 2 basic components: (1) a flexible and expandable textile suturable component that enables the implantation of the ring 


$$
\begin{aligned}
& \text { Abbreviations and Acronyms } \\
& \begin{aligned}
\text { CPB } & =\text { cardiopulmonary bypass } \\
\text { EF } & =\text { ejection fraction } \\
\text { FMR } & =\text { functional mitral regurgitation } \\
\text { ICC } & =\text { intraclass correlation coefficient } \\
\text { LV } & =\text { left ventricle } \\
\text { MA } & =\text { mitral annulus } \\
\text { MAM } & =\text { mitral annular motion } \\
\text { S-TDI } & =\text { systolic tissue Doppler imaging }
\end{aligned}
\end{aligned}
$$

according to the usual annuloplasty surgical technique and (2) an elastic component represented by a helicoid spring made of a metallic alloy.

The spring has an elastic constant of $20 \mathrm{~N} / \mathrm{m}$, corresponding to an exerted force of $160 \mathrm{~g}$ for an elongation of $20 \%$. The elastic constant of the device has been chosen empirically after animal tests and according to Literature values of annular dynamics in healthy sheep. ${ }^{3}$ The spring is circular at rest. For the implantation, the device is mounted on a holder that has the typical kidney-bean shape of the MA, with a linear anterior border (relative to the intertrigonal segment) and a circular posterior border (relative to the shape of the annulus between the commissures). When mounted on the holder, the device is loaded and is subjected to an elongation of $20 \%$. The predetermined dimension of the device, relative to the intercommissural diameter at implantation, has been derived from anatomic data from previous reports. ${ }^{2}$ Because the ring is stretched when implanted, it tends to contract the MA after implantation. Figure 1 shows the dimensions of the spring at rest and in implant configuration.

The viscoelastic properties of the annular tissue together with the left ventricular forces and pressure during the cardiac cycle determine the dynamics of the elastic device (Figure 2).

\section{Surgical Procedure}

The study was approved by the institutional ethics committee for animal research, and all animals received humane care in compliance with the "Guide for the care and use of laboratory animals" prepared by the Institute of Laboratory Animal Resources, National Research Council, and published by the National Academy Press, revised 1996. This study follows the Italian guidelines for the protection of animals used for scientific and other experimental purposes (D.L.G.S. 27/01/1992, no. 116).

Ten female Ile de France sheep, weighing $63.5 \pm 4.5 \mathrm{~kg}$ (range, 55-70 kg) and with a mean age of $18.17 \pm 6.78$ months, were anesthetized with an intravenous injection of $10 \mathrm{mg} / \mathrm{kg}$ thiopental followed by isoflurane in $100 \%$ oxygen. Mechanical ventilation was maintained with an air/oxygen mixture and a tidal volume of $20 \mathrm{~mL} / \mathrm{kg}$.

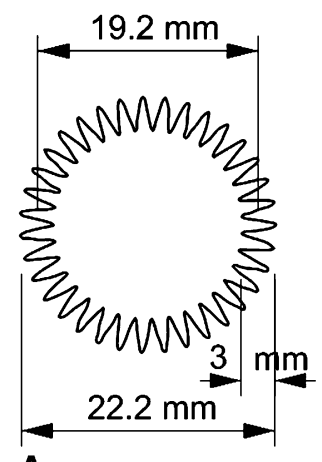

A

FIGURE 1. The elastic component of the device is represented by a helicoid spring: A, rest configuration with dimensions; B, implant configuration with dimensions.

In right lateral recumbency a left fourth intercostal thoracotomy was performed, and the heart was suspended in a pericardial cradle.

After full heparinization, normothermic cardiopulmonary bypass (CPB) was instituted, cannulating the right carotid artery with a $20 \mathrm{~F}$ cannula and the right ventricle through the pulmonary artery with a $28 \mathrm{~F}$ cannula. The pump flow was kept at a rate of $50 \mathrm{~mL} \cdot \mathrm{kg}^{-1} \cdot \mathrm{min}^{-1}$ by using a roller heart-lung machine primed with $1000 \mathrm{~mL}$ of lactated Ringer solution and bicarbonate. Blood gases were maintained in the physiologic range with a membrane oxygenator. With the heart beating, an incision on the left atrial appendage was performed, and the device was sutured to the MA by means of 10 to 12 single $\mathrm{U}$ stitches. The left atrium was closed and vented, and after a period of hemodynamic stabilization, the animals were weaned from $\mathrm{CPB}$ After the completion of angiographic and echocardiographic evaluation, the chest was closed, and the animals were weaned from mechanical ventilation and awakened.

\section{Echocardiography}

All epicardial Doppler echocardiographic studies were performed with a multifrequency transducer attached to a Vingmed System 5 (General Electric, Fairfield, Conn). All images were obtained in real time by an experienced echocardiographer in sleeping animals before and after CPB Two-dimensional echocardiographic analysis was performed epicardially through the intercostal thoracotomy and through the transdiaphragmatic approach. Long-axis, short-axis, 4-chamber, and 5-chamber views were obtained.

Three sets of measurements were made in each echocardiographic view at predetermined steps. The mean value of 3 measurements was used to describe the quantitative data for each animal at each step. End-diastolic and end-systolic measurements were obtained at the time of the peak $\mathrm{R}$-wave and the end of the T-wave, respectively.

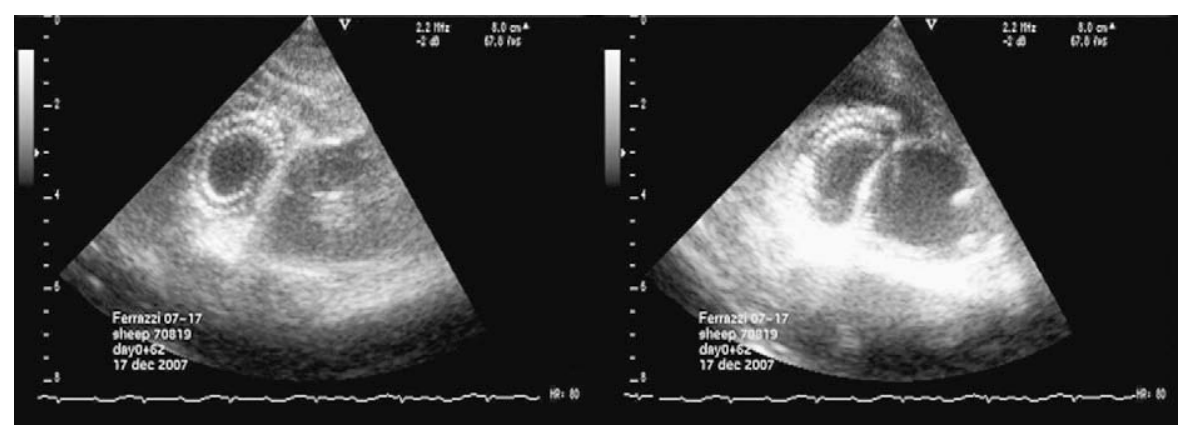

FIGURE 2. Echocardiographic imaging: change in shape and dimensions according to the cardiac cycle phases: diastole (on the left) and systole (on the right). 
Measurement of mitral annular motion (MAM), which defines the systolic movement of the MA toward the apex, was done by placing the M-mode cursor perpendicular to the lateral site of the MA (Figure 3).

Doppler tissue imaging was performed in the lateral walls with a 2-mm sampling volume by using an apical 4-chamber view. The peak of the systolic wave (systolic tissue Doppler imaging [S-TDI]) was measured. Continuous-wave Doppler scanning was used to obtain peak transmitral flow velocity (E-wave) as well as mean transmitral pressure gradient. Ejection fraction (EF) was calculated with a modified Simpson's rule. Mitral and aortic regurgitation were checked with color flow Doppler echocardiographic analysis.

\section{Angiography}

After the implantation of the mitral device, an angiographic scan was taken for each sheep. Images were acquired in the hemodynamically stable open-chest animals with a Stenoscop 9000 MDA (General Electric Medical Systems, Milwaukee, Wis) able to obtain 25 images per second. Angiography scans of the left ventricle and aorta were performed to check for the presence of mitral or aortic regurgitation. A number of angiographic sequences were taken to assess the right positioning of the mitral ring and its dynamics after implantation by using lateral and left anterior views. Data from the scans were digitized for the offline calculation of the ring area and perimeter.

Because of the radiopacity of the metallic alloy of the spring, it was possible to evaluate the spring position and dimensions.

For each sheep, at least 1 angiographic sequence was taken, relative to a frontal view of the ring. The outer border of the spring is assumed to move integrally with the MA with no relative displacement according to the technique of implantation and the device design. Each frame of the angiographic sequence was extracted and scaled by using a known reference dimension.

The contour of the spring in each frame was extracted, drawing a number of points (between 20 and 30) on the outer border. Least-squares cubic splines were drawn to join the points and create closed curves relative to the spring contour (Figure 4).

The area and perimeter of the curves were calculated during the cardiac cycle. Two different blinded operators performed the measurements using 2 different methods of segmentation of the mitral ring border: Autocad 2004 (Autodesk Systems, Alpharetta, Ga) and in-house built MATLAB code (MathWorks, Inc, Natick, Mass). The 2-dimensional area was calculated by means of the MATLAB function polyarea. The perimeter was calculated as the sum of the segments defined by the contour points.



FIGURE 3. Echocardiographic measurement of mitral annular motion (MAM).

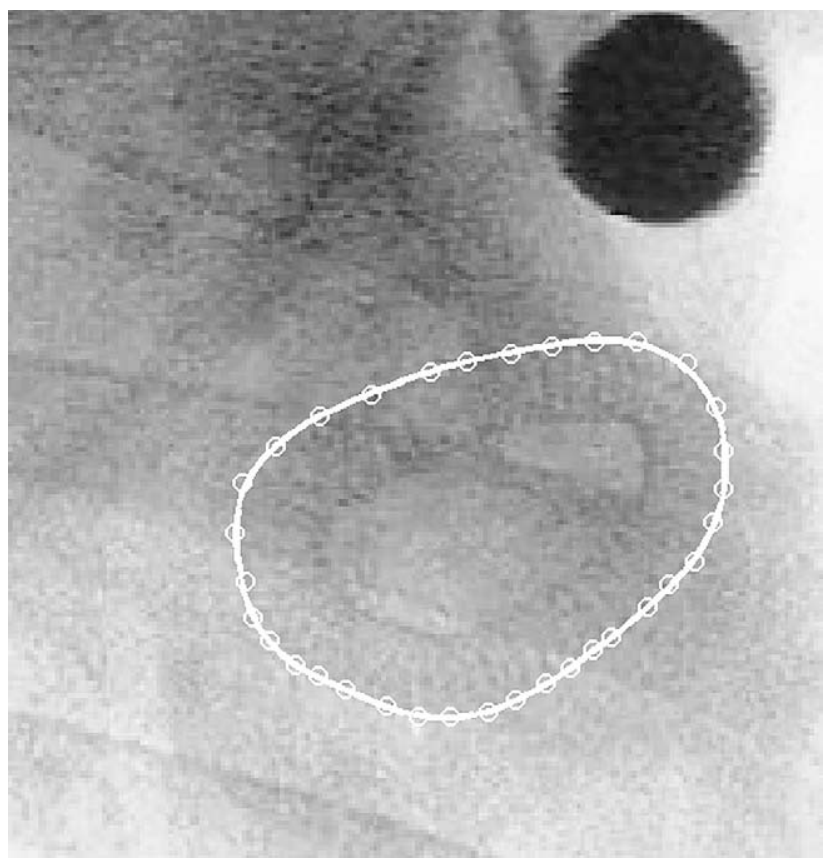

FIGURE 4. Sample of an angiographic scan of the implanted device. The white line represents the outer border, depicted to perform the measurement of the area and perimeter of the mitral annulus.

Because of the differences in heart rate for each sheep, the cardiac cycle length was normalized to 1 to allow comparison of the data. The curves were aligned, positioning the end-diastolic phase (middle point between the maximum and minimum opening of the ring) at 0.5 , according to previously reported methods. ${ }^{2,9}$

The systolic percentage of annular area and perimeter reduction was calculated for each sheep, defined as follows:

Systolic reduction $=\frac{\text { Max value }- \text { Min value }}{\text { Max value }} \cdot 100$.

\section{Statistical Analysis}

All variables were tested not to violate the normality assumption by using the Kolmogorov-Smirnov test and were reported as the mean \pm standard deviation. Comparisons between means were performed by using the paired-sample 2-sided $t$ test, as appropriate. When independent-samples $t$ tests were performed, the equality of variance between samples was assessed by using the F test. Linear correlation analysis was carried out to examine the linear relation between dependent variables. Statistical analyses were performed with the SPSS version 13.0 software package (SPSS, Inc, Chicago, Ill).

Because the offline calculation derived from the angiographic scans was performed by 2 independent operators who used 2 different methods, the measurements of systolic and diastolic area and perimeter were compared. This comparison showed a significant consistency of the measurements; for all parameters, no statistical difference was found by using the paired $t$ test and intraclass correlation coefficients (ICCs). Excellent agreement was found in case of diastolic perimeter (paired $t$ test, $P=.877$, ICC $=0.895$ ), systolic perimeter (paired t test, $P=.851, \mathrm{ICC}=0.855$ ), and diastolic area (paired $t$ test, $P=.653, \mathrm{ICC}=0.837$ ), whereas very good agreement was found in case of systolic area (paired $t$ test, $P=.500, \mathrm{ICC}=0.792$ ). ICCs proved the very high reliability of the method, yielding a value for the coefficient of greater than 0.75 for all the comparisons. 


\section{RESULTS}

Surgical procedures were carried out uneventfully for all 10 sheep. The implantation technique was simple and very close to the standard technique adopted for mitral ring annuloplasty. All the animals survived the intervention, and the acute instrumental examinations were completed in similar preimplantation and postimplantation hemodynamic conditions. All the animals were in sinus rhythm.

\section{Echocardiographic Assessment}

No mitral or aortic regurgitation was identified in any animal before and after the mitral device implantation. The systolic function, in terms of EF and S-TDI, was similar before and after implantation $\left(\mathrm{EF}_{\text {basal }}=64.5 \%, \mathrm{EF}_{\text {implantation }}=\right.$ $65.4 \%, P=.13 ; \mathrm{S}_{-\mathrm{TDI}_{\text {basal }}}=0.06, \mathrm{~S}-\mathrm{TDI}_{\text {implantation }}=0.07$, $P=.87$ ). MA plane motion was maintained after implantation, although it was reduced $\left(\mathrm{MAM}_{\text {basal }}=1.4 \mathrm{~cm}\right.$, $\left.\mathrm{MAM}_{\text {implantation }}=1.2 \mathrm{~cm}, P=.0029\right)$. Transmitral mean gradient $(P=.0026)$ and $\mathrm{E}$-wave $(P=.00092)$ increased from baseline to after implantation (Table 1).

\section{Angiographic Assessment}

All curves show similar behavior, with a maximum in the first part of the cardiac cycle (corresponding to end-diastole) and a minimum in the second part of the cardiac cycle (corresponding to early systole). Maximum (diastolic) area for the rings was $372.1 \pm 113.5 \mathrm{~mm}^{2}$, and minimum (systolic) area was $322.9 \pm 106.5 \mathrm{~mm}^{2}$; these 2 values were found to be statistically different $(P<.001)$. Maximum perimeter was $69 \pm 11.1 \mathrm{~mm}$.

The percentage of systolic annular reduction was $14.1 \%$ (range, $7.7 \%-19.7 \%$ ) in terms of area and $7.2 \%$ (range, $4.9 \%-10.0 \%$ ) in terms of perimeter. The correlation between the changes in area and perimeter was good $(r=0.77, P<.01)$. The average values for perimeter and area were calculated instant by instant over the nondimensionalized cardiac cycle for the sheep population and are plotted in Figure 5. The behavior of this curve is similar to that reported in healthy sheep by other authors. ${ }^{2,3,9}$

TABLE 1. Morphofunctional parameters: basal and postimplantation measurements

\begin{tabular}{lccc}
\hline & Basal & Postimplantation & $\boldsymbol{P}$ value \\
\hline HR (beats/min) & $96 \pm 14$ & $101 \pm 5$ & .68 \\
Systolic BP $(\mathrm{mm} \mathrm{Hg})$ & $111 \pm 18$ & $99 \pm 14$ & .20 \\
Diastolic BP $(\mathrm{mm} \mathrm{Hg})$ & $84 \pm 11$ & $78 \pm 8$ & .10 \\
EF $(\%)$ & $64.5 \pm 1.3$ & $65.4 \pm 1.6$ & .13 \\
S-TDI $(\mathrm{m} / \mathrm{s})$ & $0.06 \pm 0.01$ & $0.07 \pm 0.03$ & .87 \\
MAM $(\mathrm{cm})$ & $1.4 \pm 0.3$ & $1.2 \pm 0.3$ & .0029 \\
E $(\mathrm{m} / \mathrm{s})$ & $0.6 \pm 0.1$ & $1.2 \pm 0.2$ & .00094 \\
Transmitral $\Delta \mathrm{P}(\mathrm{mm} \mathrm{Hg})$ & $0.8 \pm 0.2$ & $3.1 \pm 1.3$ & .0026 \\
\hline
\end{tabular}

$H R$, Heart rate; $B P$, blood pressure; $E F$, ejection fraction; $S$-TDI, systolic tissue Doppler imaging wave; $M A M$, mitral annular motion; $E$, peak mitral flow velocity; transmitral $\triangle P$, mean transmitral pressure gradient.



FIGURE 5. Average area and perimeter of the outer border of the metallic spring for the animal population.

\section{DISCUSSION}

This animal study demonstrates that a fully elastic ring, implanted on the MA of a healthy sheep, allows physiologic dynamic behavior of the mitral apparatus in terms of motion of the mitral plane and variations in perimeter and area in accordance with cardiac cycle phases.

In this study, echocardiography was performed before and after the implantation of the mitral ring to assess hemodynamic and functional parameters, whereas angiography was performed only after the implantation to quantify the device's geometric changes during the cardiac cycle. All animals studied were healthy adult sheep, and no evidence of hemodynamically significant mitral regurgitation, as determined by means of Doppler echocardiographic analysis, was registered at the time of data collection before and after surgical intervention. All data were obtained with the animals ventilated and in normal sinus rhythm. Hemodynamics, including heart rate, $\mathrm{EF}$, blood pressure, and S-TDI wave, were similar before and after the implantation $(P>.1)$, enabling homogeneous observations of MA dynamics without confounding interference of LV function.

Although no undersizing of the MA was performed in this study, a certain degree of annular morphologic and dimensional changes occurred. It is well known that the implantation of any type of mitral ring reduces significantly the annular size, mainly because of the suturing technique: the use of interrupted horizontal mattress sutures, as in our experiment, placed around the entire MA contour causes a certain degree of annular stiffening and reduction. ${ }^{2}$ Because of this, some statistical differences between basal and postimplantation values of echocardiographic parameters have been registered: MAM, E-peak mitral flow velocity, and mean transmitral pressure gradient. Nevertheless, none of these parameters have crucial morphologic or hemodynamic relevance.

A major component in MA dynamics is represented by annular plane motion according to the cardiac cycle phases. ${ }^{10-12}$ The magnitude of MAM correlates with systolic and diastolic ventricular function, ${ }^{8,11}$ is subject to atriogenic 
influence, ${ }^{3,13}$ contributes to sphincteric action as an aid to ventricular filling and emptying, accounts for a relevant portion of total filling and emptying of ventricular volume, and finally determines a large amount of stroke volume. ${ }^{11}$ This MA movement is well displayed by the M-mode echocardiographic measurement of MAM. A mean MAM value of $1.2 \pm 0.3 \mathrm{~cm}$ has been registered in the present study, which confirms that a flexible and elastic device preserves annular motion, although at a reduced level. This result is similar to those reported in previous animal and clinical studies with different types of mitral annuloplasty. ${ }^{14,15}$

Several studies performed in animals and human subjects have demonstrated that annular area and perimeter are subjected to variation during the cardiac cycle in terms of dimension and shape. This phenomenon depends on systodiastolic differences in commissure-commissure and septal-lateral diameters together with the variation in curvature of the saddle shape of the annulus. ${ }^{3,16}$ The area change reported in the Literature for healthy subjects varies from $6.9 \%$ to $16.7 \%$ in ovine models $\mathrm{s}^{2,3,5,16-18}$ and from $9.3 \%$ to $26.0 \%$ in human models. ${ }^{1,19-21}$ The physiologic changes in MA area are prevalently regulated by the normal LV geometry and function. ${ }^{3,19,22}$ As reported by several authors in animal studies, ${ }^{2,18,23}$ the implantation of a complete mitral ring, no matter the type (rigid, flexible, or selectively flexible), does not allow a physiologic change in the area and perimeter of the MA. A significant annular area variation is only reported for a C-shaped annuloplasty band, which allows free motion of the intertrigonal segment of the annulus ${ }^{23}$; however, controversial results have been reported also for this type of device. ${ }^{18,23}$ In human studies mitral area variation has been reported after implantation of a flexible C-shaped band ${ }^{24}$ or flexible ring, ${ }^{25}$ whereas no significant area change was reported in case of the implantation of a rigid ring.

In our study the observed extent of ring annular area and perimeter variations were $14.1 \%$ and $7.2 \%$, respectively, suggesting that this new flexible and fully elastic ring modifies its dimensions according to the natural MA movements, allowing a physiologic value of area and perimeter variations. This result is due to the intrinsic properties of the spring.

The reduction in MA size that occurs after any ring implantation (which occurred also in our study) would likely require a greater force than that generated by the ventricular contraction to change the annular area. Following the length-tension relationship of the cardiac muscle, a shorter length of muscle fibers associated with a shorter length of the MA causes a reduction of the annular forces. ${ }^{2}$ Hence we hypothesize that the active elastic recoil force of our device might have replaced the lack of native muscular tension and therefore contributed to the physiologic variation of the annular area.

\section{Clinical Implications}

The clinical implications of this work principally refer to the field of FMR. FMR is characterized by geometric LV remodeling, with dilatation and dysfunction typically seen in idiopathic dilated cardiomyopathy and ischemic cardiomyopathy. During the last 2 decades, many experimental and clinical studies have been performed on the interpretation of the disease ${ }^{8,11,19,20,26-28}$ and the analysis of clinical results. ${ }^{5,26,29-31}$ Evolving concepts have stimulated research on the design of new generations of mitral annuloplasty devices. The principles of mitral valve repair (MVR) have evolved in an attempt to identify the best device able to cure the mitral lesion and simultaneously replicate the MA physiologic shape and dynamics. However, the intrinsic elastic properties of the normal MA, in terms of perimeter variation, area variation, folding, contraction of the posterior portion, and extension of the anterior portion, have not been taken into account. The principles that are accepted in the best practice at present refer to undersizing of the MA, reduction and fixation of septal-lateral dimension, and adoption of cause-specific geometrically shaped rings. ${ }^{29}$ This attitude appears to be a renunciation of a new thinking on FMR optimal repair that has been advocated by many authors. $3,14,16,17,19,23,32-34$

The preliminary results of our study suggest that the potential benefits of a device aimed at the correction of FMR that is flexible and fully elastic allows the physiologic dynamics of the mitral valve complex and restores elastic forces where they have been reduced because of ventricular dysfunction. ${ }^{35}$

\section{Limitations}

The presence of a metal helicoid spring constituting the device has affected the amount of information that could be extracted from echocardiographic analysis. A number of measurements of aortic and mitral diameters, as well as annular contour length, have been attempted, but the intraobserver and interobserver variability were too high to accept these measures.

Area and perimeter have been calculated by means of angiographic scans because of the radiopacity of the metal. The derived anatomic definition of the MA is more subjective than in imaging based on visually identifiable structures, such as implanted annular markers or piezoelectric crystals. ${ }^{2,3,16}$ Nevertheless, the tracing of the annular contour in the present study was performed in a consistent manner with good reproducibility, as demonstrated by the absence of significant interobserver variation. Furthermore, because no sliding between the metallic ring and the MA is present, the calculated area of the outer border of the mitral ring is proportional to the actual value of the MA area. Therefore we consider the comparison of our results with those reported by others in animal models reasonable.

Most studies on MA dynamics in animals have been performed 1 week after the implantation during mild sedation or total anesthesia. ${ }^{2,3,32}$ In our study the measurements have been performed during anesthesia and mechanical 
ventilation before chest closure. The homogeneity of preimplantation and postimplantation settings is demonstrated by the absence of a significant difference in hemodynamic parameters, and therefore the evaluation of the MA dynamics appears to be correct.

No undersizing of the MA had been planned before the study. However, all the prototypes implanted had the same size, according to the minimum value of size reported in the literature for sheep of the same weight. ${ }^{2}$ Thus a global reduction of MA might have occurred in some animals. The variation of some echocardiographic parameters registered after the implantation could have arisen from this, although it has not affected the postimplantation dynamics.

We thank Luca Corti for the technical advice he provided for the project.

\section{References}

1. Ormiston JA, Shah PM, Tei C, Wong M. Size and motion of the mitral valve annulus in man. I. A two-dimensional echocardiographic method and findings in normal subjects. Circulation. 1981;64:113-20.

2. Glasson JR, Green GR, Nistal JF, Dagum P, Komeda M, Daughters GT, et al. Mitral annular size and shape in sheep with annuloplasty rings. J Thorac Cardiovasc Surg. 1999;117:302-9.

3. Glasson JR, Komeda M, Daughters GT, Foppiano LE, Bolger AF, Tye TL, et al. Most ovine mitral annular three-dimensional size reduction occurs before ventricular systole and is abolished with ventricular pacing. Circulation. 1997;96: II1 15-22.

4. Timek TA, Dagum P, Lai DT, Tibayan F, Liang D, Daughters GT, et al. Will a partial posterior annuloplasty ring prevent acute ischemic mitral regurgitation? Circulation. 2002;106:I33-9.

5. Timek TA, Glasson JR, Lai DT, Liang D, Daughters GT, Ingels NB Jr, et al. Annular height-to-commissural width ratio of annulolasty rings in vivo. Circulation. 2005; 112:I423-8

6. Levine RA, Handschumacher MD, Sanfilippo AJ, Hagege AA, Harrigan P, Marshall JE, et al. Three-dimensional echocardiographic reconstruction of the mitral valve, with implications for the diagnosis of mitral valve prolapse. Circulation. 1989;80:589-98.

7. Jones CJ, Song GJ, Gibson DG. An echocardiographic assessment of atrial mechanical behavior. Br Heart J. 1991;65:31-6.

8. Lopez-Candales A, Rajagopalan N, Gulyasy B, Edelman K, Bazaz R. Comparative echocardiographic analysis of mitral and tricuspid annular motion: differences explained with proposed anatomic-structural correlates. Echocardiography. 2007;24:353-9.

9. Karlsson MO, Glasson JR, Bolger AF, Daughters GT, Komeda M, Foppiano LE, et al. Mitral valve opening in the ovine heart. Am J Physiol Heart Circ Physiol. 1998;274:H552-63.

10. Simonson JS, Schiller NB. Descent of the base of the left ventricle: an echocardiographic index of left ventricular function. J Am Soc Echocardiogr. 1989; 2:25-35.

11. Carlhall C, Wigstrom L, Heiberg E, Karlsson M, Bolger AF, Nylander E. Contribution of mitral annular excursion and shape dynamics to total left ventricular volume change. Am J Physiol Heart Circ Physiol. 2004;287:H1836-41.

12. Carlsson M, Ugander M, Mosen H, Buhre T, Arheden H. Atrioventricular plane displacement is the major contributor to left ventricular pumping in healthy adults, athletes, and patients with dilated cardiomyopathy. Am J Physiol Heart Circ Physiol. 2007;292:H1452-9.

13. Timek TA, Lai DT, Dagum P, Green GR, Glasson JR, Daughters GT, et al. Mitral annular dynamics during rapid atrial pacing. Surgery. 2000;128:361-7.

14. Borghetti V, Campana M, Scotti C, Domenighini D, Totaro P, Coletti G, et al. Biological versus prosthetic ring in mitral-valve repair: enhancement of mitral annulus dynamics and left-ventricular function with pericardial annuloplasty at long term. Eur J Cardiothorac Surg. 2000;17:431-9.

15. Daimon M, Gillinov AM, Liddicoat JR, Saracino G, Fukuda S, Koyama Y, et al. Dynamic change in mitral annular area and motion during percutaneous mitral annuloplasty for ischemic mitral regurgitation: preliminary animal study with realtime 3-dimensional echocardiography. J Am Soc Echocardiogr. 2007;20:381-8.

16. Lansac E, Lim KH, Shomura Y, Goetz WA, Lim HS, Rice NT, et al. Dynamic balance of the aortomitral junction. J Thorac Cardiovasc Surg. 2002;123:911-8.

17. Parish LM, Jackson BM, Enomoto Y, Gorman RC, Gorman JH 3rd. The dynamic anterior mitral annulus. Ann Thorac Surg. 2004;78:1248-55.

18. Dagum P, Timek T, Green GR, Daughters GT, Liang D, Ingels NB Jr, et al. Threedimensional geometric comparison of partial and complete flexible mitral annuloplasty rings. J Thorac Cardiovasc Surg. 2001;122:665-73.

19. Flachskampf FA, Chandra S, Gaddipatti A, Levine RA, Weyman AE, Ameling W, et al. Analysis of shape and motion of the mitral annulus in subjects with and without cardiomyopathy by echocardiographic 3-dimensional reconstruction. J Am Soc Echocardiogr. 2000;13:277-87.

20. Ahmad RM, Gillinov AM, McCarthy PM, Blackstone EH, Apperson-Hansen C, Qin JX, et al. Annular geometry and motion in human ischemic mitral regurgitation: novel assessment with three-dimensional echocardiography and computer reconstruction. Ann Thorac Surg. 2004;78:2063-8.

21. Kaplan SR, Bashein G, Sheehan FH, Legget ME, Munt B, Li XN, et al. Three-dimensional echocardiographic assessment of annular shape changes in the normal and regurgitant mitral valve. Am Heart J. 2000;139:378-87.

22. Gorman JH 3rd, Gorman RC, Jackson BM, Enomoto Y, St John-Sutton MG, Edmunds LH Jr. Annuloplasty ring selection for chronic ischemic mitral regurgitation: lessons from the ovine model. Ann Thorac Surg. 2003;76:1556-63.

23. Redmond J, Christiansen D, Bergin C, Leuer L, Ryan T, Rakow N, et al. In-vivo motion of mitral valve annuloplasty devices. J Heart Valve Dis. 2008;17:110-7.

24. Gillinov AM, Cosgrove DM 3rd, Shiota T, Qin J, Tsujino H, Stewart WJ, et al Cosgrove- Edwards Annuloplasty System: midterm results. Ann Thorac Surg. 2000;69:717-21.

25. Yamaura Y, Watanabe N, Ogasawara Y, Wada N, Kawamoto T, Toyota E, et al Geometric change of mitral valve leaflets and annulus after reconstructive surgery for ischemic mitral regurgitation: real-time 3-dimensional echocardiographic study. J Thorac Cardiovasc Surg. 2005;130:1459-61.

26. Tibayan FA, Rodriguez F, Langer F, Zasio MK, Bailey L, Liang D, et al. Annular remodeling in chronic ischemic mitral regurgitation: ring selection implications. Ann Thorac Surg. 2003;76:1549-54.

27. Watanabe N, Ogasawara Y, Yamaura Y, Wada N, Kawamoto T, Toyota E, et al Mitral annulus flattens in ischemic mitral regurgitation: geometric differences between inferior and anterior myocardial infarction: a real-time 3-dimensional echocardiographic study. Circulation. 2005;112:I458-62.

28. Kaji S, Nasu M, Yamamuro A, Tanabe K, Nagai K, Tani T, et al. Annular geometry in patients with chronic ischemic mitral regurgitation: three-dimensional magnetic resonance imaging study. Circulation. 2005;112:I409-14.

29. Fedak PW, McCarthy PM, Bonow RO. Evolving concepts and technologies in mitral valve repair. Circulation. 2008;117:963-74.

30. Carpentier AF, Lessana A, Relland JY, Belli E, Mihaileanu S, Berrebi AJ, et al The "Physioring"': an advanced concept in mitral valve annuloplasty. Ann Thorac Surg. 1995;60:1177-85.

31. Watanabe N, Ogasawara Y, Yamaura Y, Kawamoto T, Akasaka T, Yoshida K. Geometric deformity of the mitral annulus in patients with ischemic mitral regurgitation: a real-time three-dimensional echocardiographic study. J Heart Valve Dis. 2005; 14:447-52.

32. Glasson JR, Komeda MK, Daughters GT, Niczyporuk MA, Bolger AF, Ingels NB, et al. Three-dimensional regional dynamics of the normal mitral anulus during left ventricular ejection. J Thorac Cardiovasc Surg. 1996;111:574-85.

33. Dall'Agata A, Taams MA, Fioretti PM, Roelandt JR, Van Herwerden LA. Cosgrove- Edwards mitral ring dynamics measured with transesophageal threedimensional echocardiography. Ann Thorac Surg. 1998;65:485-90.

34. Okada Y, Shomura T, Yamaura Y, Yoshikawa J. Comparison of the Carpentier and Duran prosthetic rings used in mitral reconstruction. Ann Thorac Surg. 1995;59:658-62.

35. Ferrazzi P, Senni M, Iascone MR, Merlo M, Triggiani M, Lorusso R, et al. Implantation of an elastic ring at equator of the left ventricle influences cardiac mechanics in experimental acute ventricular dysfunction. J Am Coll Cardiol. 2007;50:1791-8. 